\title{
Experiencia en el uso del portafolio por medio de las redes sociales
}

\begin{abstract}
Jose-Luis Poza-Luján ${ }^{a}$, Marga Cabrera Méndezb, Miguel Rebolloc, Ángeles Calduch , Rebeca Díez-Somavillaf, Nuria Lloret Romerog, Ignacio Despujol Zabalah , Ana Albors $^{i}$ and Lola Teruel ${ }^{j}$

aEscuela Técnica Superior de Ingeniería Informática, Universitat Politècnica de València, jopolu@disca.upv.es. bEscuela Politécnica Superior de Gandia, Universitat Politècnica de València, mcabrera@upvnet.upv.es. 'Facultad de Administración y Dirección de Empresas, Universitat Politècnica de València, mrebollo@dsic.upv.es. ${ }^{\mathrm{d}}$ Escuela Técnica Superior de Ingeniería Informática Universitat Politècnica de València, mcalduch@eio.upv.es. 'Escuela Politécnica Superior de Gandia, Universitat Politècnica de València, rdiez@har.upv.es. ${ }^{\mathrm{g}}$ Escuela Técnica Superior de Ingeniería Informática, Universitat Politècnica de València, nlloret@upvnet.upv.es. 'Escuela Técnica Superior de Ingeniería del Diseño, Universitat Politècnica de València, ndespujol@asic.upv.es. ${ }^{\text {EEscuela }}$ Técnica Superior de Ingeniería Agronómica y del Medio Natural, Universitat Politècnica de València, analbors@upvnet.upv.es. jEscuela Politécnica Superior de Gandia, Universitat Politècnica de València,dteruel@upvnet.upv.es.
\end{abstract}

\begin{abstract}
At the UPV, the EICE teams: QtalNET and IEMA, are performing toghether the PIME project "Social ePortfolios", in which they are testing different assessment's techniques through electronic portfolios and social networks. The aim of the project is to develop a protocol to use social media through the process of creating e-portfolios, with the objective of value the skills acquired by students.This article describes the project and the experience consistent in various subjects, from different degrees, to evaluate the portfolios presented. Students can implement the portfolio through social networks (experimental group) or through a document (control groups). The develop is individual in every case. The weight of the portfolio in the evaluation goes from $60 \%$ to $100 \%$ (depending on the subject). The oral defense is realized to all the students (no matter if they are the experimental or the control group), they are given a brief guide with the aim of have the same structure to compare between different portfolios. In this article the method is exposed, as well as the reflections on their use.
\end{abstract}

Keywords: Portfolio, Social Network, Students evaluation. 


\begin{abstract}
Resumen
En la UPV los equipos EICE:QtalNET e IEMA realizan conjuntamente el proyecto PIME "Social ePortafolios" en el que se ensayan diferentes técnicas de evaluación por medio de portafolios electrónicos y en redes sociales.
\end{abstract}

El objetivo principal del proyecto es elaborar un protocolo que permita usar las redes sociales durante el proceso de creación de portafolios electrónico para la evaluación de las competencias adquiridas por los alumnos.

En este artículo se presenta el proyecto, así como la experiencia que se está realizando consistente en experimentar en diversas asignaturas de diferentes titulaciones el portafolios para evaluar. Los alumnos pueden implementar el portafolio a través de las redes sociales (grupos experimentales) o por medio de un documento (grupos de control). La realización en todos los casos es individual. El peso del portafolio en la evaluación va desde un 60\% a un $100 \%$ (dependiendo de la asignatura). La defensa se realiza oralmente y a todos los alumnos (tanto si son del grupo experimental como de control) se les proporciona una breve guía para que la estructura pueda ser comparable entre los diferentes portafolios. En el presente artículo se expone el método seguido así como las reflexiones sobre su uso.

Palabras clave: Portafolio, Redes Sociales, Evaluación

\title{
1. Introducción
}

En el contexto de la Universidad del siglo XXI donde la formación en competencias ha adquirido un protagonismo absoluto, la evaluación de dichas competencias, aún siendo un tema que lleva décadas de trabajos previos (Spencer et al., 1984), todavía es uno de los objetos de estudio más tratados en la docencia ya que existen una gran cantidad de método de evaluación, pero no todos proporcionan la misma información acerca de las competencias (Martínez et al., 2014).

Entre las diversas herramientas para la evaluación de competencias, el portafolio del estudiante destaca como una de las que más información proporcionan al profesor (Gil et al., 2015). El portafolio proporciona información tanto al profesor como al estudiante, ya que es una herramienta donde la reflexión del proceso de aprendizaje se une a la exposición de los resultados de la docencia (Olalla, 2015).

(c)) EY-NC-ND 2015, Universitat Politècnica de València

Congreso IN-RED (2015) 
Jose-Luis Poza-Luján, Marga Cabrera Méndez, Miguel Rebollo, Ángeles Calduch, Rebeca DíezSomavilla, Nuria Lloret Romero, Ignacio Despujol Zabala, Ana Albors and Lola Teruel

El concepto de portafolio se refiere al conjunto de evidencias (trabajos, bocetos, desarrollos y material similar) recopiladas acerca de una cuestión concreta. Generalmente en el ámbito artístico tiene mucho sentido pues permite dar a conocer la obra de dicho artista, sin embargo, de forma análoga, tiene su equivalencia en el ámbito educativo (Barberá y De Martín, 2009) donde se corresponde con una memoria documentada del proceso de aprendizaje del alumno. El portafolio puede ser tanto individual como en equipo (Pérez, 2014), aunque dependiendo de los objetivos de evaluación uno u otro método puede ser más conveniente.

La evaluación del portafolio es de por sí compleja, aunque lo más eficiente, especialmente para el alumno, es el uso de las rúbcricas (Raposo y Martínez, 2011) que permiten valorar los aspectos del portafolio que guiarán correctamente al estudiante.

Entre las herramientas para desarrollar el portafolio se encuentran los documentos clásicos, pero en los últimos tiempo, cobra especial relevancia el portafolio electrónico (Domínguez et al., 2015) especialmente por su facilidad de adaptación a diferentes modelos de enseñanza y aprendizaje (Freire et al., 2014). Dado que el uso de las redes sociales se ha extendido en los últimos años incluido en el ámbito docente (Poza et al., 2014) lo que también incluye las redes sociales como herramienta que de soporte al portafolio (Kim et al., 2010). Consiguientemente, es de gran interés y actualidad conocer cómo usar las redes sociales como medio para la evaluación de los alumnos a través del portafolio.

Basándose en todo lo anterior, los Equipos de Innovación y Calidad Educativa (EICE): Calidad en los procesos de enseñanza-aprendizaje mediante medios y redes sociales en educación universitaria (QTALNET) y Innovación en la Evaluación para la Mejora del Aprendizaje Activo (IEMA) realizan conjuntamente, dentro del programa de Proyectos de Innovación y Mejora Educativa (PIME) el proyecto "Social ePortafolios: apoyo a la evaluación con portafolios electrónicos mediante redes sociales” en el que se ensayan diferentes técnicas de evaluación por medio de portafolios electrónicos y en redes sociales y se comparan los resultados con los portafolios en papel donde se enmcuadra el presente artículo.

A continuación, en el apartado 2, se expone el objetivo principal del estudio mostrado y los objetivos operacionales en que se organiza dicho objetivo principal yque dan lugar a el desarrollo de la innovación. A continuación, en el apartado 3, se describe el desarrollo de la innovación de forma descriptiva. En el apartado 4, se muestran los ejemplos de la innovación desarrollada para pasar, en el apartado 5, a las conclusiones junto a las reflexiones correspondientes.

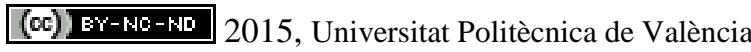

Congreso In-Red (2015) 


\section{Objetivos y objetos de estudio}

Recientemente se ha comprobado que, sin embargo, los estudiantes todavía no han asimilado las redes sociales como una herramienta de trabajo [2]. Desde el punto de vista de los autores, es posible que los alumnos todavía no vean la red social como una herramienta de trabajo, quizás porque las herramientas habituales no se han empleado todavía en el contexto de las redes sociales. Por ello, se plantea emplear herramientas docentes, concretamente el portafolio, a través de las redes sociales, para así, poder comprobar si son de utilidad para los estudiantes y les dan el valor correspondiente.

Como se mostró en la sección anterior, el portafolio puede realizarse en papel, en tinta digital o por medio de una red social (que es el caso de estudio objetivo del presente artículo). En la Tabla 1 se muestra qué tipo de material del desarrollado a lo largo del curso puede ser empleado en cada tipo de portafolio.

Tabla 1.Tipos de portafolio y material que permiten distribuir para su uso como herramienta de evaluación en la docencia universitaria

\begin{tabular}{|c|c|c|c|}
\hline $\begin{array}{c}\text { Tipo de material a } \\
\text { distribuir }\end{array}$ & $\begin{array}{l}\text { Portafolio } \\
\text { en papel }\end{array}$ & $\begin{array}{l}\text { Portafolio } \\
\text { electrónico }\end{array}$ & Portafolio en red social \\
\hline $\begin{array}{l}\text { Texto escrito: } \\
\text { reflexiones, etc. }\end{array}$ & Permitido & Permitido & Permitido \\
\hline $\begin{array}{l}\text { Imágenes estáticas: } \\
\text { fotografías, } \\
\text { infografías, etc. }\end{array}$ & Permitido & Permitido & Permitido \\
\hline $\begin{array}{l}\text { Imágenes } \\
\text { dinámicas: } \\
\text { animaciones }\end{array}$ & No permitido & $\begin{array}{l}\text { Permitido, especialmente si se } \\
\text { emplea el formato Web. No } \\
\text { permitido, o con restricciones } \\
\text { en el caso de Tinta Digital }\end{array}$ & Permitido \\
\hline Vídeos & No permitido & $\begin{array}{l}\text { Permitido en el formato Web, } \\
\text { pero con bastantes } \\
\text { restricciones en el caso de } \\
\text { tinta digital }\end{array}$ & Permitito \\
\hline Archivos & No permitido & $\begin{array}{c}\text { Permitido en el formato Web. } \\
\text { No permitido en el caso de } \\
\text { tinta digital }\end{array}$ & Permitido \\
\hline $\begin{array}{l}\text { Interacciones con } \\
\text { otros compañeros: } \\
\text { opiniones }\end{array}$ & No permitido & $\begin{array}{l}\text { Permitido en formato Blog, } \\
\text { pero con un tratamiento } \\
\text { distintivo y con poca } \\
\text { simultaneidad }\end{array}$ & $\begin{array}{l}\text { Permitido con tratamiento } \\
\text { igualitario en el caso de todos } \\
\text { los usuarios del grupo de la } \\
\text { red social }\end{array}$ \\
\hline
\end{tabular}


Jose-Luis Poza-Luján, Marga Cabrera Méndez, Miguel Rebollo, Ángeles Calduch, Rebeca DíezSomavilla, Nuria Lloret Romero, Ignacio Despujol Zabala, Ana Albors and Lola Teruel

A partir del planteamiento expuesto anteriormente se construye el objetivo principal del artículo: exponer la experiencia en el uso del portafolio, ya sea en formato electrónico como por medio de las redes sociales, como herramienta de evaluación y de reflexión en el proceso de enseñanza-aprendizaje en el ámbito universitario.

Para alcanzar el objetivo principal se han planteado los siguientes objetivos operacionales:

- Emplear las redes sociales, complementando al Learning Management System (LMS) institucional, para trabajar la asignatura (o alguno de sus aspectos: teoría, seminarios o prácticas).

- Desarrollar un portafolio electrónico basándose en el trabajo realizado a través de las redes sociales que permita realizar parte de la evaluación de la asignatura.

- Realizar una encuesta al alumnado implicado para conocer la visión que éstos han tenido de la experiencia.

- Analizar los resultados, tanto de la encuesta al alumnado como de los propios portafolios, para desarrollar métodos que permitan el uso de las redes sociales como soporte en el uso de portafolios en la docencia universitaria.

Los objetivos expuestos anteriormente dan lugar al desarrollo de la innovación expuesto en el siguiente apartado. Cabe destacar que lo que se pretende es emplear aquellos ítems mostrados en la Tabla 1 en los que las redes sociales permiten ofrecer un material diferenciado sobre los otros medios. Se debe aclarar que el resultado del objetivo no es demostrar que las redes sociales sean mejores que otros medios para implementar un portafolio sino ofrecer una vía de evaluación diferenciada, y posiblemente complementaria, a los otros métodos.

\section{Desarrollo de la innovación}

El proyecto define dos grupos de trabajo: el grupo de control, consistente en los grupos en los que ya se ha experimentado el portafolio por parte del equipo IEMA, y dos grupos experimentales, uno de ellos realizará portafolios electrónicos documentados mediante una memoria en archivo pdf y otro de ellos empleará la red social Facebook para desarrollar el portafolio. En el caso del grupo experimental que emplea Facebook, se ha creado un grupo por asignatura para la gestión de los portafolios (y de otros aspectos relacionados con la asignatura). Los resultados obtenidos por ahora se contextualizan en diversas asignaturas de máster, donde, por medio de eventos privados o de documentos privados, los alumnos gestionan su portafolio. La puntuación del portafolio varía dependiendo de la asignatura entre el $60 \%$ y el $100 \%$ de la nota final.

En el caso presentado en esta comunicación, la forma de funcionamiento se muestra en la Figura 1. Facebook permite la realización de grupos de usuarios relacionados con un tema concreto (Park et al., 2009). Los grupos de Facebook se vienen empleando en algunas 
Universidades como vehículo de comunicación docente (Bosch, 2009) con mostrada eficiencia en los aspectos docentes (Meishar et al., 2012). Los grupos pueden ser públicos (cualquiera puede leer los comentarios y saber quiénes pertenecen al grupo), privados (sólo pueden ver los comentarios los componentes del grupo, pero cualquiera puede ver quién pertenece al grupo) y secretos (el grupo no puede ser localizado en Facebook). Este aspecto es importante puesto que se les preguntó a los alumnos acerca de las preferencias, siendo el método privado el más aceptado. En todos los tipos de grupos hay una serie de administradores que se encargan de su gestión, especialmente en el aspecto de la admisión de componentes.

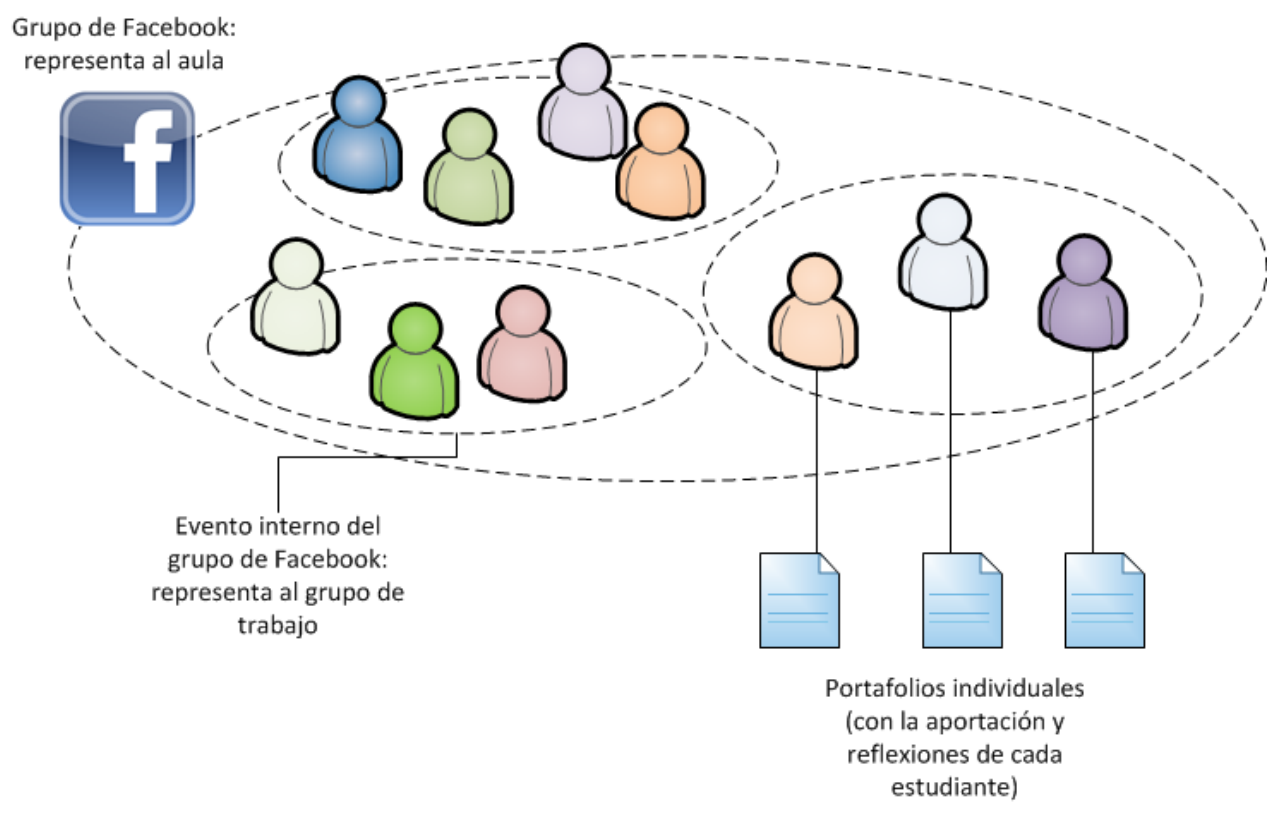

Fig. 1 Estructura de la organización del grupo de Facebook para la creación del portafolio.

Como puede verse en la Figura 1, Facebook no permite grupos dentro de grupos, cosa que sí que permiten otras redes como LinkedIn. Dado que la mayoría de los alumnos prefirieron Facebook, los grupos de alumnos se gestionaron por medio de eventos donde la fecha de vencimiento del evento es la fecha de la evaluación de la asignatura. Además, los eventos de los grupos de trabajo son privados, siendo el profesor y los componentes de cada grupo de trabajo los únicos que pueden ver las publicaciones, permitiendo evitar que "copien” los alumnos entre ellos y separando la información común (dudas, notificaciones, etc.) en el muro del grupo y la información de cada grupo de trabajo (desarrollos, códigos de programación, etc.) en el muro del evento privado.

En esta comunicación se presenta el caso concreto de la asignatura de "Automatización Distribuida" perteneciente al segundo cuatrimestre del primer curso del "Máster en 
Jose-Luis Poza-Luján, Marga Cabrera Méndez, Miguel Rebollo, Ángeles Calduch, Rebeca DíezSomavilla, Nuria Lloret Romero, Ignacio Despujol Zabala, Ana Albors and Lola Teruel

Mecatrónica” de la Escuela Técnica Superior de Ingeniería del Diseño (ETSID) de la UPV. La asignatura tiene un total de 4.5 créditos, siendo principalmente práctica. La experiencia se ha desarrollado en el curso 2014-2015 con un número total de 32 alumnos matriculados de los cuales, 26 escogieron la modalidad de portafolio desarrollado en Facebook. En el portafolio deben de incluir el trabajo práctico de la asignatura, ya que los aspectos teóricos de conocimiento y comprensión son evaluados mediante un test al final de cada unidad temática. Por lo tanto el portafolio, en este caso, se refiere exclusivamente a las competencias prácticas (análisis, diseño y aplicación de sistemas de automatización). Los alumnos que no escogieron el portafolio en red social realizan su trabajo individualmente. En todos los casos, cada alumno debe entregar una memoria, pero los que han desarrollado su trabajo práctico mediante un grupo de trabajo de Facebook, pueden emplear el mismo en la exposición y hacer referencia a todas las publicaciones en la Red Social, es decir, su historial de publicaciones en el evento es su portafolio digital siendo la memoria únicamente la reflexión individual.

La evaluación del portafolio se realiza mediante una entrevista personal con el profesor, de diez minutos (son 32 alumnos) en la que el alumno expone el portafolio al profesor. En el caso de la asignatura presentada el portafolio es un $60 \%$ de la nota final. Todos los alumnos disponen de una propuesta de esquema de portafolio:

1. Introducción

1.1. Descripción del proyecto

1.2. Objetivos

1.3. Descripción del documento

2. Diseño

2.1. Especificación conceptual (dibujos)

2.2. Especificación formal (esquemas y diagramas de estados)

2.3. Dificultades encontradas y cómo se han solucionado

3. Implementación

3.1. Prototipos desarrollados

3.2. Código desarrollado

3.3. Dificultades encontradas y cómo se han solucionado

4. Evaluación

4.1. Pruebas realizadas

4.2. Dificultades encontradas y cómo se han solucionado

5. Conclusiones

5.1. Aportaciones del proyecto

5.2. Ampliaciones

Además de la orientación de los contenidos, los alumnos también disponían de la rúbrica de evaluación que se muestra en la Tabla 2.

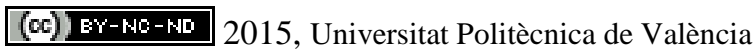

Congreso In-Red (2015) 
Experiencia en el uso del portafolio por medio de las redes sociales

Tabla 2. Rúbrica empleada en la evaluación del portafolios

\begin{tabular}{|c|c|c|c|c|c|}
\hline $\begin{array}{l}\text { Aspecto a } \\
\text { evaluar }\end{array}$ & $\begin{array}{c}\% \\
\text { Nota }\end{array}$ & $\begin{array}{l}\text { 9+ } \\
\text { Excelente }\end{array}$ & $\begin{array}{c}\text { 7-8 } \\
\text { Bueno }\end{array}$ & $\begin{array}{l}\text { 5-6 } \\
\text { Suficiente }\end{array}$ & $\begin{array}{c}\quad<5 \\
\text { Insuficiente }\end{array}$ \\
\hline Contenido & $50 \%$ & $\begin{array}{l}\text { Incluye planos } \\
\text { precisos y fotos } \\
\text { adecuadas de los } \\
\text { montajes en amplia } \\
\text { cantidad. Todos los } \\
\text { documentos de } \\
\text { apoyo son } \\
\text { relevantes. Detalla } \\
\text { de forma óptima las } \\
\text { aportaciones al } \\
\text { proyecto de grupo. } \\
\text { Los contenidos de } \\
\text { opinión y/o materia } \\
\text { son precisos, } \\
\text { adecuados y muy } \\
\text { elaborados }\end{array}$ & $\begin{array}{l}\text { Incluye planos } \\
\text { precisos y fotos } \\
\text { adecuadas. Todos } \\
\text { los documentos de } \\
\text { apoyo son } \\
\text { relevantes. Detalla } \\
\text { la aportación al } \\
\text { proyecto de } \\
\text { grupo, l. Los } \\
\text { contenidos de } \\
\text { opinión y/o } \\
\text { materia son } \\
\text { precisos y } \\
\text { adecuados }\end{array}$ & $\begin{array}{l}\text { Incluye planos y fotos } \\
\text { aunque con carencias. } \\
\text { Los documentos de } \\
\text { apoyo se ajustan al } \\
\text { mínimo exigido. } \\
\text { Cuenta la aportación al } \\
\text { proyecto de grupo. } \\
\text { Llos contenidos de } \\
\text { opinión y/o materia } \\
\text { están presentes pero } \\
\text { poco desarrollados o } \\
\text { poco claros }\end{array}$ & $\begin{array}{l}\text { No se incluyen } \\
\text { planos ni fotos o su } \\
\text { cantidad es } \\
\text { extremadamente } \\
\text { limitada. No se } \\
\text { aportan los } \\
\text { suficientes } \\
\text { documentos de } \\
\text { apoyo. No se detalla } \\
\text { la aportación al } \\
\text { proyecto del grupo. } \\
\text { los contenidos de } \\
\text { opinión y/o materia } \\
\text { son irrelevantes o no } \\
\text { están incluidos. }\end{array}$ \\
\hline $\begin{array}{l}\text { Organización } \\
\text { y conexión de } \\
\text { ideas }\end{array}$ & $25 \%$ & $\begin{array}{l}\text { La organización y } \\
\text { conexión son } \\
\text { apropiadas. }\end{array}$ & $\begin{array}{l}\text { Organización } \\
\text { controlada. } \\
\text { conexión con } \\
\text { pocos problemas }\end{array}$ & $\begin{array}{l}\text { La organización está } \\
\text { parcialmente } \\
\text { controlada; la } \\
\text { conexión a veces está } \\
\text { ausente o es parcial. }\end{array}$ & $\begin{array}{l}\text { Hay poca o ninguna } \\
\text { organización y } \\
\text { conexión aparente. }\end{array}$ \\
\hline $\begin{array}{l}\text { Precisión } \\
\text { técnica }\end{array}$ & $15 \%$ & $\begin{array}{l}\text { Los códigos } \\
\text { funcionan } \\
\text { adecuadamente. La } \\
\text { selección de } \\
\text { componentes es la } \\
\text { adecuada al } \\
\text { proyecto. }\end{array}$ & $\begin{array}{l}\text { Los códigos } \\
\text { funcionan } \\
\text { razonablemente } \\
\text { bien. La selección } \\
\text { de componentes } \\
\text { es relativamente } \\
\text { precisa y se } \\
\text { adaptan al } \\
\text { proyecto. }\end{array}$ & $\begin{array}{l}\text { Los códigos funcionan } \\
\text { suficientemente } \\
\text { bien.La selección de } \\
\text { componentes son poco } \\
\text { precisas y poco } \\
\text { adecuadas al proyecto. }\end{array}$ & $\begin{array}{l}\text { Los códigos no } \\
\text { funcionan. La } \\
\text { selección de } \\
\text { componentes es } \\
\text { incorrecta e } \\
\text { inadecuadas al } \\
\text { proyecto. }\end{array}$ \\
\hline $\begin{array}{c}\text { Formato } \\
\text { (portada, } \\
\text { índice, } \\
\text { estructura } \\
\text { numérica, } \\
\text { bibliografía) } \\
\text { ortografía, } \\
\text { puntuación, } \\
\text { longitud, citas }\end{array}$ & $10 \%$ & $\begin{array}{l}\text { El formato es } \\
\text { impecable y } \\
\text { atractivo. No hay } \\
\text { errores de ortografía } \\
\text { y puntuación. La } \\
\text { longitud es } \\
\text { adecuada. Las } \\
\text { fuentes se citan } \\
\text { correctamente. }\end{array}$ & $\begin{array}{l}\text { El formato es } \\
\text { correcto. Los } \\
\text { errores de } \\
\text { puntuación y de } \\
\text { ortografía no } \\
\text { distraen del } \\
\text { conjunto. Las } \\
\text { fuentes se citan } \\
\text { correctamente. }\end{array}$ & $\begin{array}{l}\text { Los errores de } \\
\text { formato, ortografía y } \\
\text { puntuación en } \\
\text { ocasiones distraen la } \\
\text { atención del conjunto. } \\
\text { Las fuentes a veces no } \\
\text { se citan } \\
\text { correctamente. }\end{array}$ & $\begin{array}{l}\text { Los errores en } \\
\text { formato, ortografía, } \\
\text { puntuación y citación } \\
\text { de fuentes son } \\
\text { inaceptables. }\end{array}$ \\
\hline
\end{tabular}

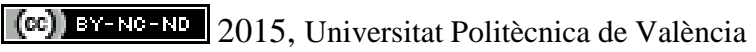


Jose-Luis Poza-Luján, Marga Cabrera Méndez, Miguel Rebollo, Ángeles Calduch, Rebeca DíezSomavilla, Nuria Lloret Romero, Ignacio Despujol Zabala, Ana Albors and Lola Teruel

\section{Resultados}

Una vez puesto en funcionamiento el grupo de Facebook, los alumnos se dispusieron a trabajar las sesiones de laboratorio. En la Figura 2 se muestra la portada del grupo con un ejemplo de evento al que los alumnos se unen para asistir a una sesión de prácticas adicional.

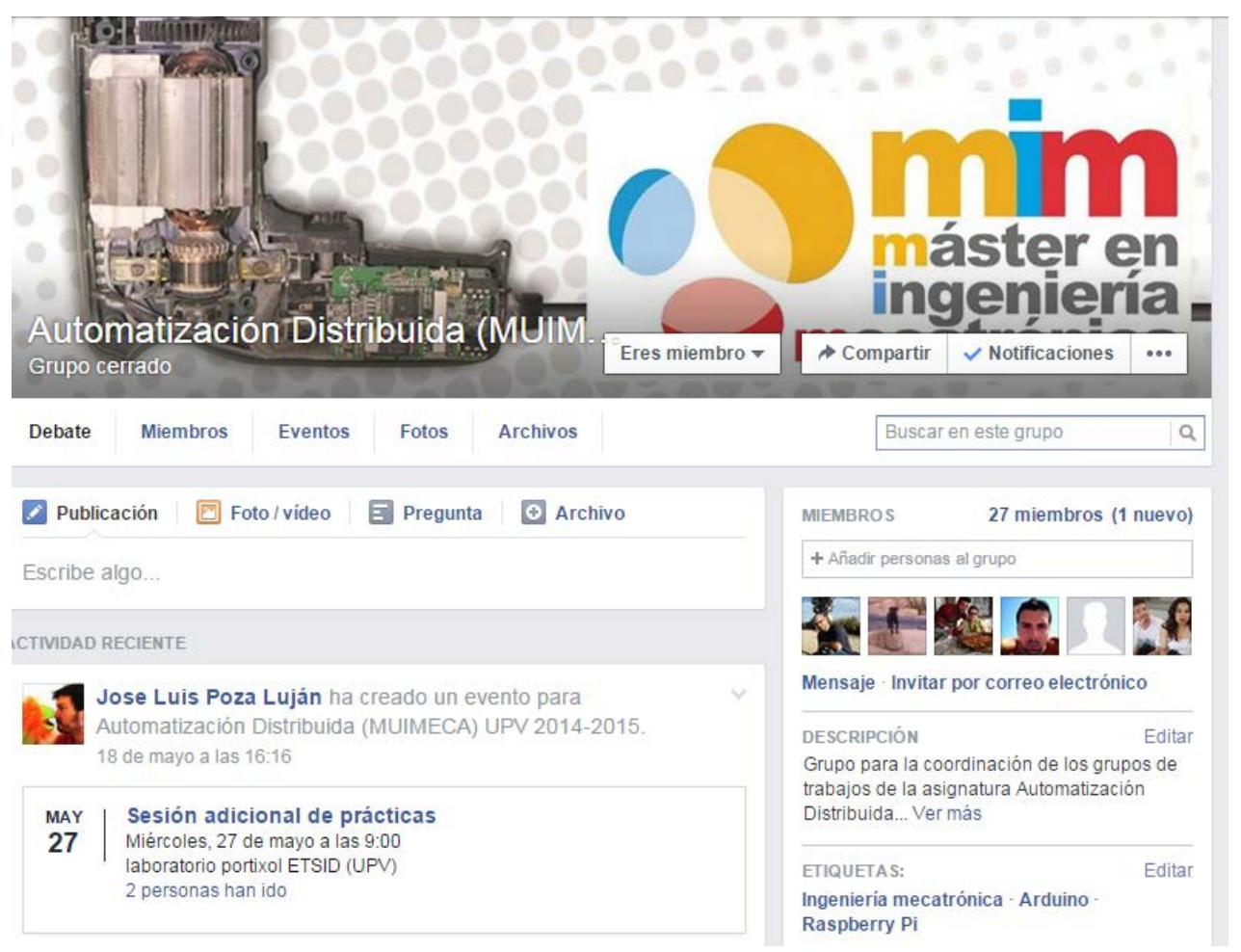

Fig. 2 Portada del grupo de Facebook con el que se ha hecho la experiencia

El grupo de Facebook, funciona como un Learning Management System (LMS) al estilo de los corporativos, como PoliformaT, empleado en la UPV (Roldán et al., 2006), pero con un aspecto y dinamismo mejorado. Los eventos del grupo privado (Figura 3) representan los grupos (en la figura sólo hay un subconjunto) de trabajo de los alumnos. El hecho de tener la opción de incluir una imagen y un nombre al evento hace que los alumnos se "diviertan" en este aspecto. 
Experiencia en el uso del portafolio por medio de las redes sociales

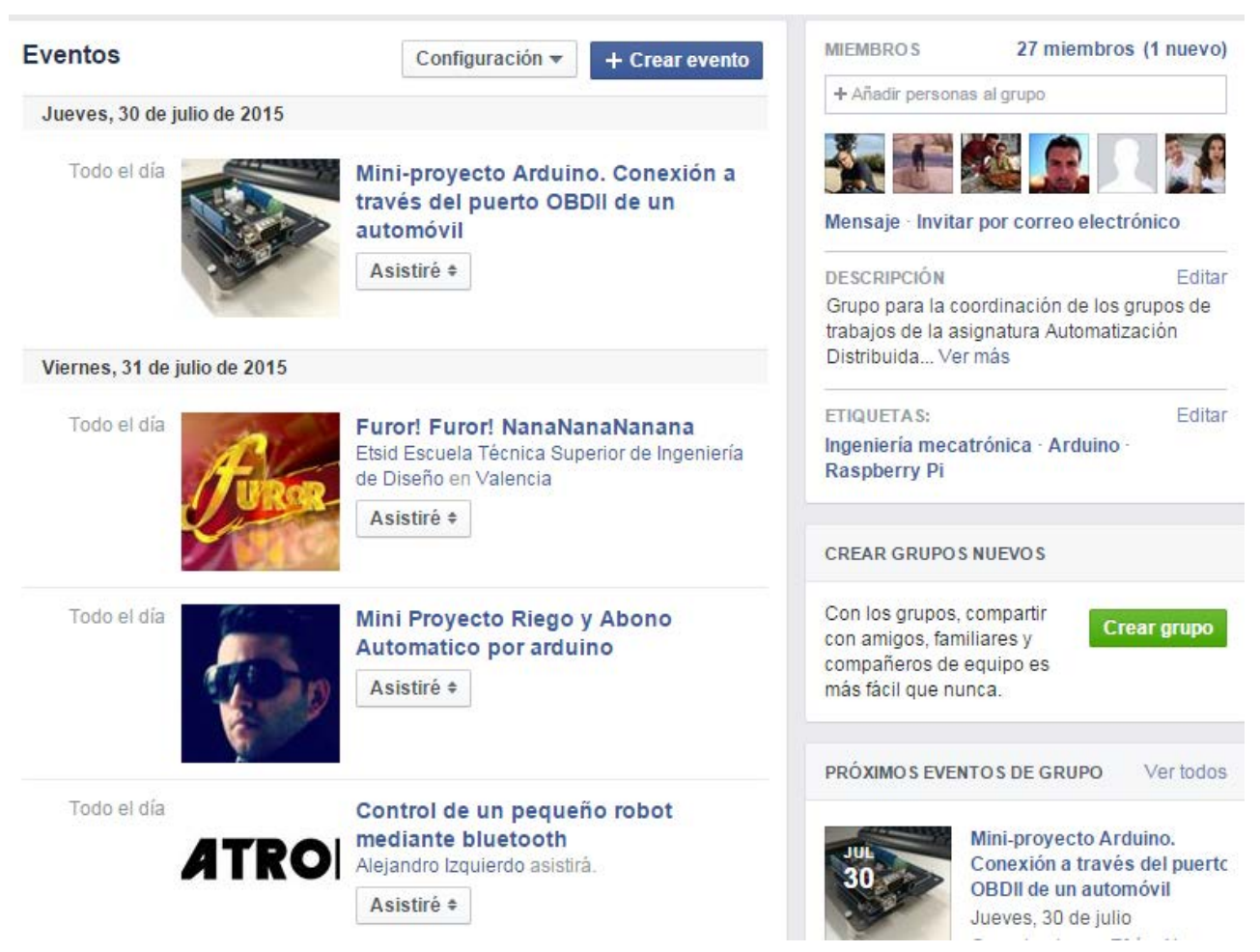

Fig. 3 Captura de pantalla de los eventos empleados para la gestión de los grupos de trabajo.

Dentro de cada evento, los alumnos van incluyendo diverso material, vídeos de los prototipos, montajes electrónicos, dudas, etc. Un ejemplo del muro de un grupo de trabajo puede verse en la Figura 4. 
Jose-Luis Poza-Luján, Marga Cabrera Méndez, Miguel Rebollo, Ángeles Calduch, Rebeca DíezSomavilla, Nuria Lloret Romero, Ignacio Despujol Zabala, Ana Albors and Lola Teruel

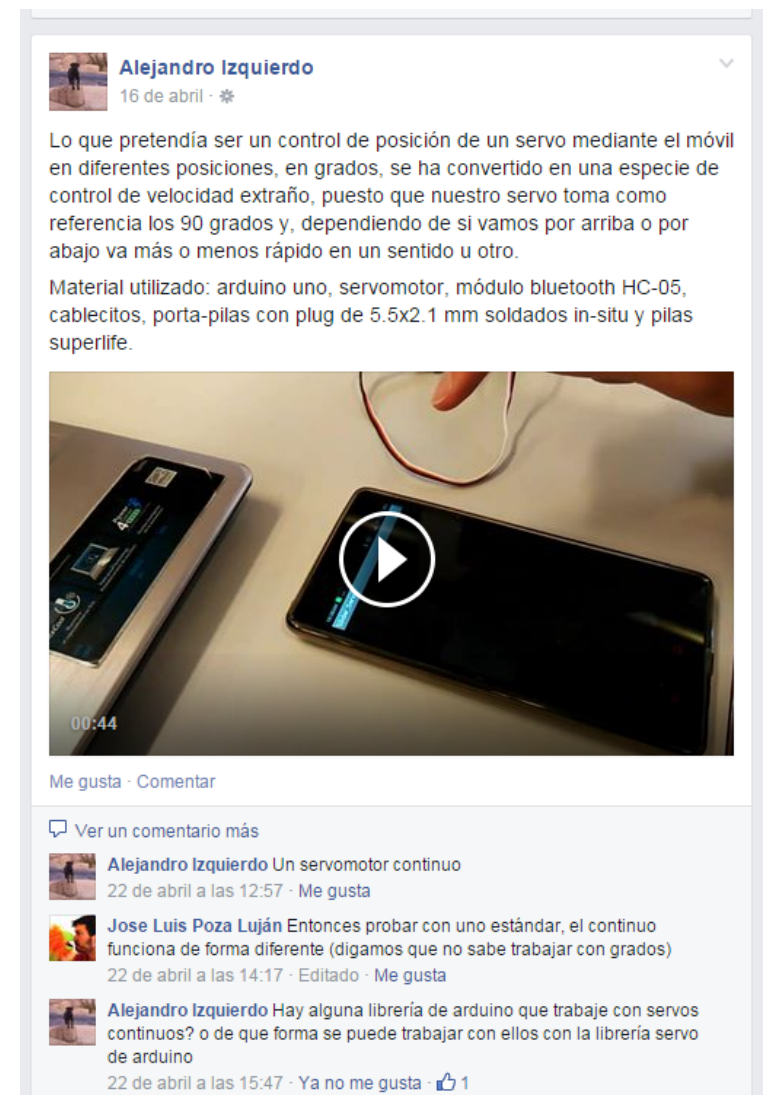

Fig. 4 Captura de pantalla de un vídeo de prueba empleado por un alumno para consultar una duda dentro del evento de un grupo de trabajo.

Como puede verse en la figura 4, el muro del evento se emplea, principalmente, para la resolución de dudas y para la muestra del avance realizado por los componentes del grupo. Es de un gran interés este aspecto, puesto que esos vídeos (la selección que el alumno haga de los más destacados), serán parte del portafolio que deberá presentar al profesor.

\section{Conclusiones}

Como primera experiencia cabe destacar como aspectos más relevantes la eficiencia en la comunicación que la red social proporciona. El grupo de Facebook no suple al LMS corporativo, sino que lo complementa. Los alumnos ven en PoliformaT la forma "oficial” donde hacen sus exámenes, disponen de los apuntes de la asignatura o reciben las comunicaciones oficiales. Sin embargo, el grupo de Facebook es considerado un medio “informal” donde poder hablar más distendidamente, intercambiar opiniones, etc.

\section{(c)) EY-NC-ND 2015, Universitat Politècnica de València}


En el caso concreto de la evaluación, a falta de disponer de las notas finales, los alumnos aprecian considerablemente que un evento de Facebook pueda ser el soporte electrónico de su portafolio, ya que el evento lo van enriqueciendo de información a medida que avanza el curso, por lo que al final el portafolio sólo es un paso casi automático que sirve para lo que ha sido diseñado: síntesis y reflexión acerca del trabajo realizado.

Como aspectos no tan positivos se debe destacar que la gestión de un grupo de Facebook implica un trabajo adicional para el profesor, ya que éste es un dinamizador en este tipo de entornos sociales. Éste aspecto debería ser considerado como actividad docente. También se debe destacar que el hecho de que Facebook sea un medio asociado a la persona y no a la institución hace que algunos alumnos sean reticentes a emplearlo. Como es lógico estas reticencias están justificadas y deben ser tenidas en consideración. Sin embargo, el hecho de no usar la red social hace que la evaluación, aunque sea la misma (entrevista con el alumno para revisar el portafolio) puede suponer que hay una vía diferenciada de evaluación.

Sin embargo, el dinamismo que proporciona el uso de grupos de redes sociales, en nuestro caso Facebook, es muy positivo, los alumnos que participan ven en la red social una forma de promocionar sus avances, compartir dudas y soluciones con sus compañeros. Cuando a este grupo, o evento, se le añade la evaluación por medio del portafolio, se dota de una seriedad al grupo, lo que implica que el alumno considera que el grupo se ha convertido un una extensión del aula.

Es de destacar que, como ampliaciones al trabajo presentado, se debe comprobar cómo incide en las notas de los alumnos, cosa que se espera poder comprobar en sucesivos estudios. Además la opinión del alumnado con respecto al método empleado también debe ser considerada, no todos quieren que un medio, como Facebook, que emplean en su vida personal, sea también un medio de trabajo.

\section{Referencias}

Barberà Gregori, Elena y de Martín Rojo, Elena (2009) Portfolio electrónico: aprender a evaluar el aprendizaje. Editorial UOC. Barcelona,

Bosch, T. E. (2009). Using online social networking for teaching and learning: Facebook use at the University of Cape Town. Communicatio: South African Journal for Communication Theory and Research, 35(2), 185-200.

Domínguez-García, S., García-Planas, M. I., Martín, R. P., \& Torres, J. T. (2015). Uso del e-portafolio en la formación: el e-portafolio integral. Revista del Congrés Internacional de Docència Universitària i Innovació (CIDUI). 
Jose-Luis Poza-Luján, Marga Cabrera Méndez, Miguel Rebollo, Ángeles Calduch, Rebeca DíezSomavilla, Nuria Lloret Romero, Ignacio Despujol Zabala, Ana Albors and Lola Teruel

Freire, F. O., Fernández, R. L., Álvarez, D. L., Álvarez, E. L., Álvarez, W. L., \& Fernández, R. A. (2014). Sistema de evaluación docente mediante el modelo 360 grados y el portafolio electrónico. Medisur, 12(1), 334-339.

Gil, M. G., Nieto, J. E. S., \& Ramos, A. D. (2015). The Students Portfolio as an Alternative Strategy to the Standardized Competencies Assessment Tests. Qualitative Research in Education, 4(1), 71-101.

Kim, P., Ng, C. K., \& Lim, G. (2010). When cloud computing meets with Semantic Web: A new design for e-portfolio systems in the social media era. British Journal of Educational Technology, 41(6), 1018-1028.

Martinez, M., Olmedo, N., Amante, B., Farrerons, O., \& Cadenato, A. (2014). Analysis of Assessment Tools of Engineering Degrees. INTERNATIONAL JOURNAL OF ENGINEERING EDUCATION, 30(6), 1689-1696.

Meishar-Tal, H., Kurtz, G., \& Pieterse, E. (2012). Facebook groups as LMS: A case study. The International Review of Research in Open and Distributed Learning, 13(4), 33-48.

Olalla, A. G. (2015). El portafolio docente. Un instrumento para evaluación y mejora de la práctica docente. Revista del Congrés Internacional de Docència Universitària i Innovació (CIDUI), (2).

Park, N., Kee, K. F., \& Valenzuela, S. (2009). Being immersed in social networking environment: Facebook groups, uses and gratifications, and social outcomes. CyberPsychology \& Behavior, 12(6), 729-733.

Pérez, I. P. (2014). El trabajo en equipo mediante el uso del portafolio y las rúbricas de evaluación: innovación en la enseñanza universitaria. REIRE: revista d'innovació i recerca en educació, 7(1), 56-75.

Poza-Lujan, J. L., Calduch-Losa, Á., Albors, A., Cabrera, M., Teruel, D., Rebollo, M., \& Somavilla, R. D. (2014). Propuesta de parámetros y caracterización de los grupos de las redes sociales orientados a la docencia universitaria: experiencia y resultados. RED. Revista de Educación a Distancia, (44), 88-105.

Poza-Lujan, J-L., Marga Cabrera, Ángeles Calduch-Losa, Rebeca Díez y Nuria Lloret. Experiencias de docencia apoyada en redes sociales en la Universidad Politécnica de Valencia. En Cabrera Méndez, M., \& Díez Somavilla, R. (2015). Congreso Internacional sobre redes sociales. COMUNICA2.

Raposo, M., \& Martínez, E. (2011). La rúbrica en la enseñanza universitaria: Un recurso para la tutoría de grupos de estudiantes. Formación universitaria, 4(4), 19-28.

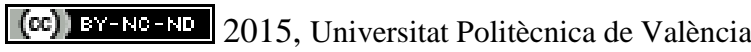

Congreso In-Red (2015) 
Experiencia en el uso del portafolio por medio de las redes sociales

Roldán, D., Ferrando, M., Busquets, J., \& Mengod, R. (2006). Software libre en la Educación Superior: PoliformaT, la plataforma de e-learning de la UPV. In Conocimiento Abierto, Sociedad Libre. III Congreso Online del OCS.

Spencer, L. M., McClelland, D. C., \& Spencer, S. M. (1994). Competency assessment methods: History and state of the art. Hay/McBer Research Press.

\section{Agradecimientos}

Los autores deseamos agradecer su apoyo a la Escuela Técnica Superior de Ingeniería Informática (ETSINF), al Departamento de Informática de Sistemas y Computadores (DISCA) de la Universitat Politécnica de València (UPV) por su apoyo. El presente trabajo está realizado bajo el programa de Proyectos de Innovación y Mejora Educativa (PIME) el proyecto "Social ePortafolios: apoyo a la evaluación con portafolios electrónicos mediante redes sociales” sustentado por el Insitituto de Ciencias de la Educación (ICE) de la UPV. 